\title{
On the Accuracy of a Precipitation Coverage Index Computed from Radar Reports
}

\author{
James T. Bunting And John H. Conover \\ Air Force Cambridge Research Laboratories, Bedford, Mass. \\ (Manuscript received 21 July 1970, in revised form 2 November 1970)
}

\begin{abstract}
The Radar Index (RI) is presented as a statistic used to study convective activity in the summer monsoon over southeast Asia. The RI is defined simply as the percent coverage of echo within $50 \mathrm{n}$ mi of the radar. A computer technique is described which accepts coded radar reports (RAREPS) as input, calculates the RI, and plots the original RAREP. Hourly values of the RI have been calculated for four summers in southeast Asia.

This approach to radar data has a number of limitations. The technique is critically examined by comparing values of the RI computed from RAREPS with those found directly from PPI scope photography and also with hourly rainfall data. The RI estimates the probability of rain better than the total rainfall. The major drawback of this technique is inaccurate RAREPS. The major advantages are application to areas with limited rainfall data and the speed with which months of data can be processed on a computer.
\end{abstract}

\section{Introduction}

In studies of clouds and weather over southeast Asia during the summer monsoon (Conover, 1967), an index of convective activity on the synoptic scale was needed. While such indices could be developed from rainfall measurements, raingage data in southeast Asia can be misleading since they are scattered and usually do not provide hourly rainfall measurements. Furthermore, at the onset of these studies, rainfall data were scarce and often not collected for months. Therefore, in view of the urgency of the work, attention was turned to the new weather radars in southeast Asia and from them Conover (1967) developed a method to reduce each hourly radar report (RAREP) to one number, a precipitation coverage index, which was referred to as the Radar Index (RI). This index is similar to the parameter "proportional area covered by echoes" which was determined quantitatively by Kessler and Russo (1963) and used with other pattern dimensions in their studies

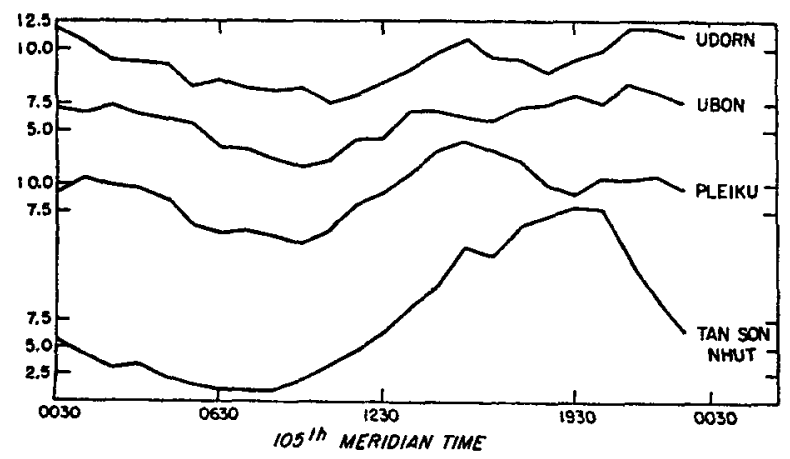

FrG. 1. The average diurnal variation of the Radar Index at four stations for June, July and August 1967. of statistical properties of weather radar echoes measured during four storms.

The purpose of this paper is to explain the Radar Index, describe its limitations, and assess its accuracy with the view that others may be interested in the use of the same or similar types of index. Variations of the index in southeast Asia are illustrated but details showing some applications of the RI in weather studies are reported elsewhere (Conover, 1970). The index has been used operationally as a predictand in objective forecasts of weather activity in southeast Asia.

\section{The Radar Index}

The RI is defined simply as the percent coverage by echoes within a circle having a $50 \mathrm{n}$ mi radius centered at the radar. Longer radii may be used; however, our studies are concerned with activity closer to the station. The RI is computed from coded hourly RAREPS, in which the radar observer approximates echoes on the PPI scope as polygons for irregular groups of echoes, thin rectangles for lines of echoes, and small circles for individual echoes, and locates each area in polar coordinates. Each of these areas is described as widely scattered $(5 \%$ covered), scattered $(25 \%)$, broken $(70 \%)$, solid $(95 \%)$, or as a cell $(100 \%)$. The percent figures were assigned after the observations were taken but are consistent with formal RAREP procedures as given by the Air Weather Service (1967). For example, a radar operator is instructed to report as widely scattered an area with related or similar echoes covering less than $10 \%$. We arbitrarily assume that all such areas have $5 \%$ coverage. A computer program was written to calculate hourly values of the RI using coded RAREPS as input (Kelley, 1968). The RAREPS are punched on 
cards, one line per card, in a format very similar to the original coding used by the radar operators. For each echo area, the part within $50 \mathrm{n} \mathrm{mi}$ of the radar is computed first. Generally speaking, the problem to be solved concerns a large circle around the radar partially overlapped by small circles, rectangles, and arbitrary and irregular polygons. Hence, the area to be calculated is bounded by straight lines and arcs of a circle. An elaborate geometrical algorithm is required to compute areas within the circle. Once these areas are found, the program then multiplies each area by the appropriate percent, adds these results and divides by the total area of the circle to yield the RI. To simplify checking, the input data for each RAREP may be drawn by an automatic plotter to simulate the appearance of the PPI scope as approximated by the radar operator. Missing echoes or improperly oriented echoes are then easily noticed.

\section{RI variation in southeast Asia}

The RI has been computed since 1966 for the months June through September when the southwest monsoon brings widespread convective precipitation to this area. RAREPS are available from a 3-cm CPS-9 radar at Tan Son Nhut, South Vietnam, and 10-cm WSR-57 radars at Pleiku, South Vietnam, and Ubon and Udorn, Thailand. Diurnal tendencies of the RI at these four radars are plotted in Fig. 1. As can be seen Pleiku, Ubon and Udorn have activity distributed fairly evenly throughout the day with some tendency for a semidiurnal variation. In contrast, the Tan Son Nhut radar has a distinct RI maximum in the late afternoon to early evening. Tan Son Nhut, only $25 \mathrm{n}$ mi from the coast, apparently has a diurnal RI variation determined by a sea breeze effect. Analysis of the hourly frequency of echoes in the horizontal reveals the general tendency for echoes to move inland and intensify during the day. The center of activity moves back toward the sea later at night while weakening. Fig. 2 is a more complete representation of the time variation in the RI at Tan Son Nhut. Time of day increases from left to right, while the date increases from top to bottom. Again, the RI tends to be higher in the late afternoon and early evening hours; however, the highest values are found in periods of several successive days and are not scattered randomly. Further, the high values of RI often tend to spill over into the early hours of the following day. This figure illustrates the problems of forecasters in southeast Asia. During the summer monsoon there is considerable variation in activity as seen in the RAREPS not only during the day but also from day to day.

\section{Limitations to the RI}

The RI uses only a small part of the potential information in a RAREP. The regulations specify that observers not only approximate the areal distribution of echoes but also record the heights, intensities, tendencies of intensities, and movements of echoes or groups of echoes out to a distance of $200 \mathrm{n}$ mi or more from the radar. The regulations place great emphasis on identifying echoes associated with severe weather. On the other hand, our method considers only the areal extent of echoes within $50 \mathrm{n} \mathrm{mi}$ of the radar. Consequently, the RI lacks information on the vertical extent or intensity of echoes, and gives no clue as to the size distribution of individual echoes.

The accuracy of the RI depends on the ability of radar operators to estimate the coverage of precipitation echoes on the PPI scope. We are uncertain how well the radar operators define echoes in the presence of ground clutter, anomalous propagation, and extreme attenuation. Also, we expect some error in approximating the shape of radar echoes and assigning weights (scattered, broken, etc.) to them.

In order to better understand how well echoes are routinely approximated by observers, and thereby assess the accuracy of RI values in the tropics, a small

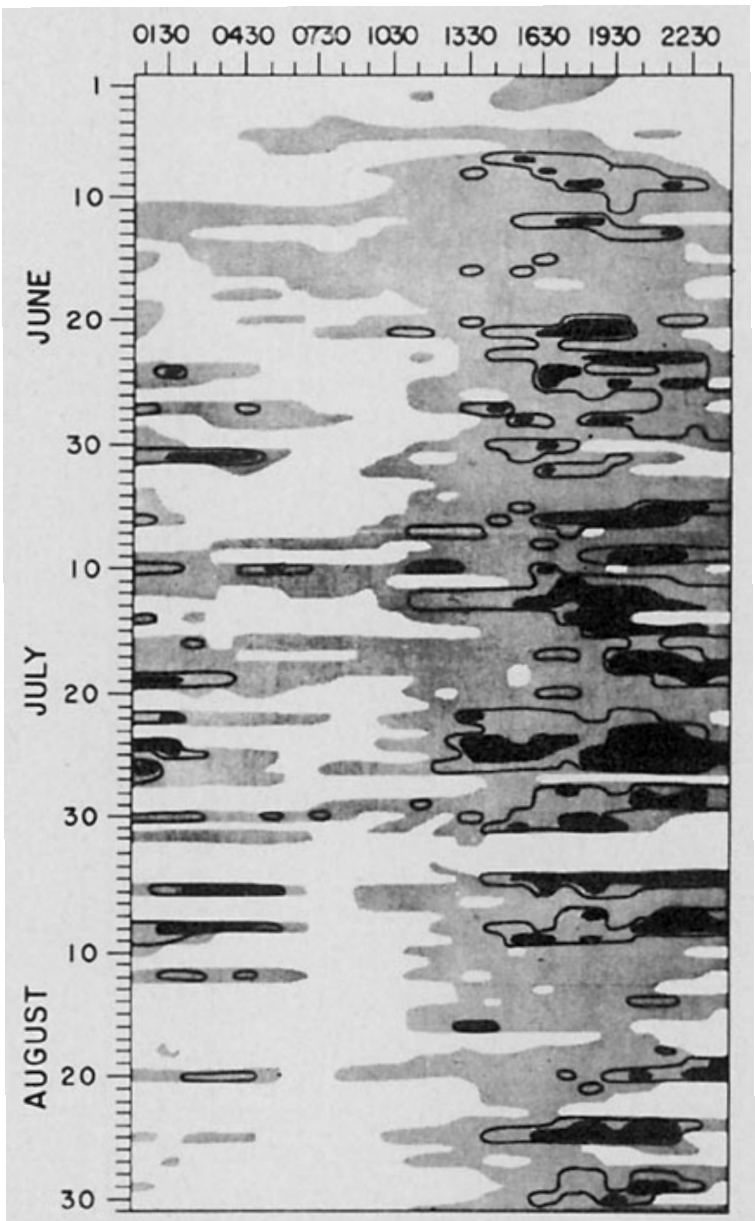

FIG. 2. Hourly (local times) and daily variations of the Radar Index at Tan Son Nhut during the summer months of 1967. The RI values run from $0.1-15$ for the lightest shade of grey, from $15.1-30$ for the intermediate shade (contoured), from $30.1-45$ for the darker shades, to $>45.1$ for black. 


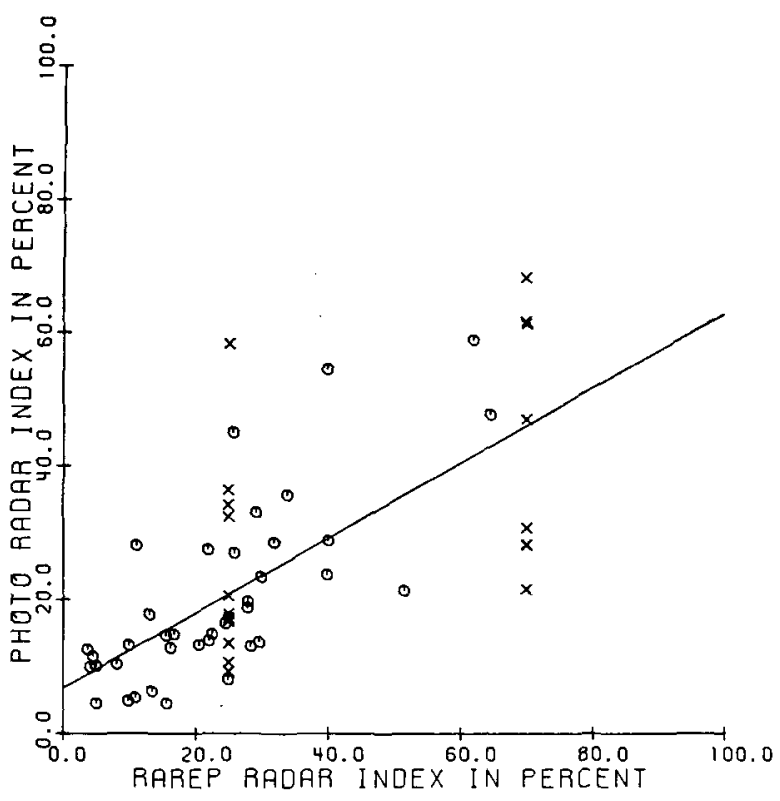

FIG. 3. A comparison of RI values calculated from RAREPS with values determined from photographs of the PPI scope using the Miami WSR-57 radar. Cases plotted with an $X$ have a RAREP RI value of exactly $25 \%$ or $70 \%$.

study was made comparing RAREPS to PPI scope photography for the WSR -57 radars at Ubon and Udorn, and at Miami, Fla. All cases were from the summer months and are believed to be free of anomalous propagation or any other suspicious ground clutter. Values of the RI were computed from the RAREPS, while the corresponding photographs were planimeterized to find the percent coverage of echoes within $50 \mathrm{n}$ mi of the radar.

Comparison of hourly values of RI measured from photographs and the corresponding values calculated from RAREPS yielded correlation coefficients of 0.69 for 60 cases at Miami, 0.63 for 113 cases at Ubon, and 0.66 for 150 cases at Udorn. Fig. 3 shows the data for Miami. For each case plotted, the $x$ axis gives the RI as determined from the RAREP while the $y$ axis gives the RI as determined from PPI scope photography. There is a great deal of scatter about the leastsquares regression line. Values plotted with an $X$ deserve special comment. These have a RAREP RI value of exactly $25 \%$ or $70 \%$, and occur when the whole 50 $\mathrm{n}$ mi circle is enclosed by an echo area labeled scattered or broken. It is obvious that there will be little precision in the values of RI which we compute if echo areas are drawn this large.

In order to test whether or not different operators might take RAREPS differently, an analysis of variance was done on the differences between the RAREP RI and the photography RI for six operators at Ubon. One operator underestimated the echo coverage by an average of $2 \%$ of the $50 \mathrm{n} \mathrm{mi} \mathrm{circle,} \mathrm{while} \mathrm{other} \mathrm{operators}$ overestimated by averages as great as $8 \%$. Using the $F$ distribution, the averages for the six operators are found to be different at the $1 \%$ significance level. These results suggest a further limitation in our method of obtaining the RI from RAREPS since it does not consider radar operators individually.

In fairness to the operators who took the RAREPS, it should be noted that the photographs are not necessarily an accurate and consistent representation of the echo coverage. Their exposure can be poor, and, at least in our study, some timing errors are possible. We have not attempted to estimate the uncertainty in the values of RI measured from photographs.

In light of these limitations to RI hourly values, time averages were used in -our applications to index and forecast the convective activity in southeast Asia. For the most part, averages are taken over the twelve afternoon hours, although we sometimes use 3- or 24-hr averages. Another approach is to rank days according to their RI averages, and divide the list into three tertiles. Once this is done, the synoptic conditions on the high average days can be compared to conditions on the low average days.

\section{Suggested changes for RAREP procedures}

Changes in regulations and guidelines for taking RAREPS that would insure a more accurate RI are:

1) Place restrictions on the maximum size of areas approximating groups of echoes. Presently, an operator can draw one large area with a low weighting factor such as scattered or widely scattered when, in fact, several smaller areas with higher weighting factors would more accurately represent the true echo coverage.

2) Allow more flexibility for weighting factors. The factors "scattered" and "broken," to which we assign the arbitrary figures 25 and $70 \%$, include all coverage densities from 10 to $90 \%$. A better approach would be to instruct the radar operators to estimate the density of coverage in echo areas to the nearest $10 \%$.

3) Code RAREPS strictly according to procedures. Abbreviations, omissions, and other departures from coding rules make RAREPS unusable in our computer program.

\section{RI correlations with rainfall data}

Values of the RI can be compared directly with rainfall measurements. While it would be impractical to establish a raingage network in southeast Asia, some data are available from scattered stations. Unfortunately, the limited information in the RI does not permit the results of many years of research in radarrainfall relationships to be applied directly. Most of this latter research has involved data obtained through photography or special signal analyzing equipment, considering the intensity of the return signal, and making assumptions as to the size distribution of scatterers. If and when operational data and techniques of this type become available, a more complicated Radar Index 
can be defined and more precise measurements can be made.

Hourly rainfall data are available at Tan Son Nhut for the 1966-68 summer months. The correlation coefficient of average daily $\mathrm{RI}$ with the total daily rainfall is only 0.35 (244 cases). When the number of hours having a trace or more of rain is compared to the average daily RI, the correlation coefficient is found to have the more significant value, 0.72 . This correlation is 0.68 for the number of hours having $0.1 \mathrm{~mm}$ or more of rain instead of hours of any rain, and decreases to 0.58 for the number of hours having $0.5 \mathrm{~mm}$ or more of rain. Since higher correlation coefficients are associated with the hours of rain rather than amounts of rain, this aspect of the comparison was expanded to include three more stations, all within $50 \mathrm{n} \mathrm{mi} \mathrm{of} \mathrm{Tan} \mathrm{Son} \mathrm{Nhut.} \mathrm{All}$ four stations had completed WBAN forms which contain hourly observations, for the summer months of 1966. To determine the number of hours of rain, we counted the number of hourly observations in which rain of any intensity was observed. When the total number of hours with rain for the four stations is compared to the average daily RI, the correlation is 0.82 (71 cases). This result substantiates the view that the RI represents the frequency, or probability, of rain better than the amount of rainfall.

\section{Conclusions}

Values of the RI do provide fairly good estimates of the probability of rain within a specified area centered at the radar, and are thereby useful in representing the degree of disturbed weather in the tropics. Over similar areas the index estimates rainfall amount poorly. The chief reason for an imperfect relationship between rain occurrence and the RI index is the inaccuracy of esti- mates of echo coverage. In the present regulations for taking RAREPS, echo areas can be made too large, while the choice of weighting factors such as scattered, broken, etc., is too small.

On the other hand, a very large number of RAREPS, which can be processed by our method, are stored at the National Weather Records Center. They have the advantage of the experience of the radar operator in distinguishing anomalous propagation and other ground clutter from meteorological echoes. With a computer, one can process one month of RAREPS in a reasonably short run to obtain insight concerning weather activity, especially where raingages are scattered or missing altogether.

A more desirable index would include the intensity and vertical extent of echoes. Such an index cannot be accurately generated from present RAREPS. As an alternative to RAREPS, careful photography or special analyzing hardware, which were not permitted on the southeast Asia radars, would, of course, be more useful.

\section{REFERENCES}

Air Weather Service, 1967: Radar weather observations. Manual 105-27, U. S. Air Force.

Conover, J. H., 1967 : Studies of clouds and weather over Southeast Asia utilizing satellite data. First Progress Rept., Satellite Meteorology Branch, Air Force Cambridge Research Laboratories, Bedford, Mass.

- 1970: Summer monsoon studies of clouds and weather over Southeast Asia utilizing satellite data. Proc. Symp. Tropical Meleorology, Honolulu, Amer. Meteor. Soc., D IX-1-D IX-6.

Kelley, R. G., 1968: Design of a digital simulation of the polar planimeter for calculating areas of echoes from radar weather observations. Sci. Rept. No. 2, Contract F19628-67-C-0125, Air Force Cambridge Research Laboratories, Bedford, Mass.

Kessler, E., and J. A. Russo, 1963: Statistical properties of weather radar echoes. Proc. 10th Wea. Radar Conf., Washington, D. C., Amer. Meteor. Soc., 25-33. 